\title{
Termites of the West Indies, the Bahamas, and Bermuda
}

\section{Thomas E. Snyder ${ }^{1}$ \\ INTRODUCTION}

Based on specimens in the collection of the U.S. National Museum, and on authoritative records in literature, the following lists have been compiled of the species of termites known to be present in Bermuda, the Bahamas, the Greater Antilles and the Lesser Antilles of the West Indies, Curacao, and Trinidad. The first list is a systematic arrangement of termites by species; the second list is by islands, giving all the records from each locality. Identifications based on nymphs being very difficult, and for some forms impossible, the concluding keys to the species from these islands are based on the soldiers and winged adults.

\section{TERMITES OF BERMUDA, THE BAHAMAS, AND THE WEST INDIES}

\section{Listed by Species}

KALOTERMITIDAE

Kalotermes approximatus Snyder: Bermuda.

$K$. bequaerti Snyder: Bermuda, Cuba, St. Croix, St. Thomas.

$K$. cubanus Snyder: Cuba.

$K$. incisus Silvestri: Barbados, Mona Island, St. Croix.

$K$. jouteli Banks: Bahamas (Andros Island), Cuba.

$K$. milleri Emerson: Jamaica.

K. mona Banks: Mona Island.

$K$. schwarzi Banks: Cuba, Jamaica, New Providence (Nassau).

$K$. snyderi Light: Bermuda, Bimini-Bahamas, Cuba, Mona Island, Puerto Rico, St. Croix.

Neotermes castaneus (Burmeister): Barbados, Cuba, Dominica, Haiti, Jamaica, Montserrat, Puerto Rico, Trinidad.

Neotermes n. sp.: Jamaica.

$N$. holmgreni Banks: Tobago, Trinidad.

Procryptotermes corniceps (Snyder): Puerto Rico, Mona Island.

Procryptotermes n. sp.: Jamaica.

Cryptotermes brevis (Walker): Barbados, Cuba, Curacao, Dominica, Dominican Republic, Grenada, Guadeloupe, Haiti, Jamaica, Puerto Rico, St. Croix, St. Kitts, St. Lucia, St. Thomas, Tobago, Trinidad.

${ }^{1}$ Retired. Formerly with the Division of Forest Insect Investigations, Bureau of Entomology and Plant Quarantine, Agricultural Research Administration, USDA, Washington, D. C. 
C. cavifrons Banks: Bermuda, Cuba, Haiti, New Providence (Nassau), Puerto Rico, St. Croix.

C. dudleyi Banks: Trinidad.

C. havilandi (Sjostedt): Barbados.

Glyptotermes liberatus (Snyder): Jamaica.

G. posticus (Hagen): Cuba, Jamaica, St. Thomas.

G. pubescens Snyder: Puerto Rico.

Calcaritermes nigriceps (Emerson): Trinidad.

\section{RHINOTERMITIDAE}

Heterotermes cardini (Snyder): Bahamas (Andros Island) Nassau, Cuba, Dominican Republic, Haiti.

H. convexinotatus (Snyder): Barbados, Cuba, Haiti, Jamaica, Puerto Rico, St. Croix, St. Thomas.

H. tenuis (Hagen): Bahamas, Cuba, Dominica, Guadeloupe, Haiti, Jamaica, Montserrat, Puerto Rico, Tobago, Trinidad.

Coptotermes havilandi Holmgren: Barbados (introduced), Jamaica (introduced).

C. testaceus (Linnaeus): Tobago, Trinidad.

Prorhinotermes simplex (Hagen): Cuba, Jamaica, Puerto Rico.

Rhinotermes marginalis (Linnaeus): Dominican Republic, Haiti, Martinique.

Dolichorhinotermes longilabius (Emerson): Trinidad.

TERMITIDAE

Anoplotermes banksi Emerson: Trinidad.

A. brevipilis Emerson: Trinidad.

A. meridianus Emerson: Dominican Republic, Haiti, Martinique, Puerto Rico.

A. schwarzi Banks: Cuba.

A. spp.: Haiti, Puerto Rico, Trinidad.

Speculitermes silvestrii (Emerson): Trinidad.

Microcerotermes arboreus Emerson: Haiti, Puerto Rico, Tobago, Trinidad.

$M$. exiguus (Hagen): Trinidad.

Crepititermes verruculosus (Emerson): Trinidad.

Termes hispaniolae (Banks): Cuba, Haiti, Isle of Pines, Jamaica, Tobago, Trinidad.

T. panamaensis (Snyder): St. Croix, Trinidad.

Cavitermes tuberosus (Emerson): Trinidad.

Neocapritermes angusticeps (Emerson): Trinidad.

Nasutitermes costalis (Holmgren): Antigua, Barbados, Cuba, Dominica, 
Dominican Republic, Grenada, Guadeloupe, St. Lucia, St. Kitts, Tobago, Trinidad, Haiti, Jamaica, Martinique, Puerto Rico, St. Croix.

$N$. ephratae (Holmgren): Guadeloupe, Montserrat, Tobago, Trinidad.

$N$. guayanae (Holmgren): Trinidad.

N. hubbardi Banks: Cuba, Jamaica.

$N$. intermedius (Banks): Trinidad.

$N$. lividus (Burmeister): Cuba, Dominican Republic, Haiti.

$N$. nigriceps (Haldeman): Curacao, Jamaica, Puerto Rico, St. Croix, St. Thomas, San Salvador, Trinidad, Vieques Island (near Puerto Rico).

$N$. rippertii (Rambur): Bahamas (Andros Island, Bimini, New Providence, Nassau; Dry Key, and Mangrove Key), Cuba, Eleuthera, Jamaica.

Parvitermes brooksi (Snyder): Biminis, Bahamas; Cuba.

$P$. discolor (Banks): Cuba, Culebra Island, Puerto Rico.

$P$. flaveolus (Banks): Haiti.

$P$. pallidiceps (Banks): Haiti.

$P$. wolcotti (Snyder): Puerto Rico.

Obtusitermes aequalis (Snyder): Cuba.

Velocitermes antillarum (Holmgren): Dominican Republic, Haiti.

V. toussainti (Banks): Dominican Republic, Haiti.

Labiotermes labralis, subsp. boreus (Emerson): Trinidad.

Armitermes holmgreni Snyder: Trinidad.

Angularitermes nasutissimus (Emerson): Trinidad.

Subulitermes baileyi (Emerson): Trinidad.

S. parvellus (Silvestri): Trinidad.

S. snyderi (Emerson): Trinidad.

S. spp.: Trinidad.

\section{Listed by Localities}

Bermuda

KALOTERMITIDAE.-Kalotermes approximatus Snyder, $K$. bequaerti Snyder, $K$. snyderi Light, Cryptotermes cavifrons Banks.

Bahamas, Andros

KALOTERMITIDAE.-Kalotermes jouteli Banks. RHINOTERMitidae.-Heterotermes cardini (Snyder), H. tenuis (Hagen). TERMITIDAE.-Nasutitermes rippertii (Rambur).

\section{Bimini}

KALOTERMITIDAE.-Kalotermes snyderi Light.

TERMirmdat- Nasutitermes rippertii (Rambur), Parvitermes brooksi (Snyder). 
Dry Key, Eleuthera, Mangrove Key TERMITIDAE.-Nasutitermes rippertii (Rambur).

New Providence (Nassau)

Kalotermitidae.-Kalotermes schwarzi Banks, Cryptotermes cavifrons Banks.

RHINOTERMITIDAE.-Heterotermes cardini (Snyder), H. tenuis (Hagen). TERMITIDAE. - Nasutitermes rippertii (Rambur).

\section{Antigua}

TERMTIDAE.-Nasutitermes costalis (Holmgren).

Barbados

KALOTERMITIDAE.-Kalotermes incisus Silvestri, Neotermes castaneus (Burmeister), Cryptotermes brevis (Walker), C. havilandi (Sjostedt).

RHINOTERMITIDAE.-Heterotermes convexinotatus (Șnyder), Coptotermes havilandi Holmgren.

TERMITIDAE. - Nasutitermes costalis (Holmgren).

\section{Cuba}

KAlotermitidat.-Kalotermes bequaeri $i$ Snyder, $K$. cubanus Snyder, $K$. jouteli Banks, $K$. schwarzi Banks, $K$. snyderi Light, Neotermes castaneus (Burmeister), Cryptotermes brevis (Walker), C. cavifrons Banks, Glyptotermes posticus (Hagen).

RHINOTERMITIDAE.-Heterotermes cardini (Snyder), H. convexinotatus (Snyder), H. tenius (Hagen), Prorhinotermes simplex (Hagen).

TERMITIDAE.-Anoplotermes schwarzi Banks, Termes hispaniolae Banks, Nasutitermes costalis (Holmgren), N. hubbardi Banks, N. lividus (Burmeister), N. rippertii (Rambur), Parvitermes brooksi (Snyder), $P$. discolor (Banks), Obtusitermes aegualis (Snyder).

\section{Culebra}

TERMITIDAE.-Parvitermes discolor (Banks).

\section{Curacao}

KALOTERMITIDAE.-Cryptotermes brevis (Walker).

TERMITIDAE.-Nasutitermes nigriceps (Haldeman).

\section{Dominica}

KALOTERMITIDAE.-Neotermes castaneus (Burmeister), Cryptotermes brevis (Walker). RHINOTERMITIDAE.-Heterotermes tenuis (Hagen). TERMITIDAE.-Nasutitermes costalis (Holmgren). 
Dominican Republic

KALOTERMITIDAE.-Cryptotermes brevis (Walker).

RHINOTERMITIDAE.-Heterotermes cardini (Snyder), Rhinotermes marginalis (Linnaeus).

TERMITIDAE.-Anoplotermes meridianus Emerson, Nasutitermes costalis (Holmgren), N. lividus (Burmeister), Velocitermes antillarum (Holmgren), $V$. toussainti (Banks).

\section{Grenada}

KALOTERMITIDAE.-Gryptotermes brevis (Walker). TERMITIDAE.-Nasutitermes costalis (Holmgren).

\section{Guadeloupe}

KALOTERMITIDAE.-Cryptotermes brevis (Walker). RHINOTERMITIDAE.-Heterotermes tenuis (Hagen).

TERMITIDAE.-Nasutitermes costalis (Holmgren), Nasutitermes ephratae (Holmgren).

\section{Haiti}

KALOTERMITIDAE.-Neotermes castaneus (Burmeister), Cryptotermes brevis (Walker), C. cavifrons Banks, Glyptotermes posticus (Hagen).

RHINotermitidat.-Heterotermes cardini (Snyder), $H$. convexinotatus (Snyder), $H$. tenuis (Hagen), Rhinotermes marginalis (Linnaeus).

TERMiTIDas.-Anoplotermes meridianus Emerson, Anoplotermes spp., Microcerotermes arboreus Emerson, Termes hispaniolae (Banks), Nasutitermes costalis (Holmgren), N. lividus (Burmeister), Parvitermes flaveolus (Banks), P. pallidiceps (Banks), Velocitermes antillarum (Holmgren), $V$. toussainti (Banks).

Isle of Pines

TERMTIDAE.-Termes hispaniolae (Banks).

Jamaica

Kalotermitidae.-Kalotermes milleri Emerson, $K$. schwarzi Banks, Neotermes castaneus (Burmeister), Neotermes n. sp., Procryptotermes n. sp., Cryptotermes brevis (Walker), Glyptotermes liberatus (Snyder), G. posticus (Hagen).

RHINOTERmitidae.-Heterotermes convexinotatus (Snyder), $H$. tenuis (Hagen), Coptotermes havilandi Holmgren, Prorhinotermes simplex (Hagen). iERMITIDAE.-Termes hispaniolae (Banks), Nasutitermes costalis (Holmgren), $N$. hubbardi Banks, N. nigriceps (Haldeman). 


\section{Martinque}

RHINOTERMITIDAE.-Rhinotermes marginalis (Linnaeus).

TERMITIDAE.-Anoplotermes meridianus Emerson, Nasutitermes costalis (Holmgren).

\section{Mona}

KALOTERmitidae.-Kalotermes incisus Silvestri, $K$. mona Banks, $K$. snyderi Light, Procryptotermes corniceps (Snyder).

\section{Montserrat}

KALOTERMITIDAE.-Neotermes castaneus (Burmeister). RHINOTERMITIDAE.-Heterotermes tenuis (Hagen). TERMITIDAE.-Nasutitermes ephratae (Holmgren).

\section{Puerto Rico}

KALOTERmitidae.-Kalotermes snyderi Light, Neotermes castaneus (Burmeister), Procryptotermes corniceps (Snyder), Cryptotermes brevis (Walker), C. cavifrons Banks, Glyptotermes pubescens Snyder.

RHINOTERMITIDAE.-Heterotermes convexinotatus (Snyder), H. tenuis (Hagen), Prorhinotermes simplex (Hagen).

TERMITIDAE.-Anoplotermes meridianus Emerson, Microcerotermes arboreus Emerson, Nasutitermes costalis (Holmgren), N. nigriceps (Haldeman), Parvitermes discolor (Banks), P. wolcotti (Snyder).

St. Kitts (St. Christopher)

KALOTERMITIDAE.-Cryptotermes brevis (Walker). TERMITIDAE.-Nasutitermes costalis (Holmgren).

\section{St. Lucia}

KALOTERMITIDAE.-Cryptotermes brevis (Walker). TERMITIDAE.-Nasutitermes costalis (Holmgren).

San Salvador (Watling)

TERMTTIDAE.-Nasutitermes nigriceps (Haldeman).

\section{Tobago}

KALOTERMITIDAE.-Neotermes holmgreni Banks, Cryptotermes brevis (Walker).

RHINOTERMITIDAE.-Heterotermes tenuis (Hagen), Coptotermes testaceus (Linnaeus).

TERMITIDAE.-Microcerotermes abroreus Emerson, Termes hispaniolae (Banks), Nasutitermes costalis (Holmgren), N. ephratae (Holmgren). 


\section{Trinidad}

KALOTERMITIDAE.-Neotermes castaneus (Burmeister), N. holmgreni Banks, Cryptotermes brevis (Walker), C. dudleyi Banks; Calcaritermes nigriceps (Emerson).

RHINOTERMITIDAE.-Heterotermes tenuis (Hagen), Coptotermes testaceus (Linnaeus), Dolichorhinotermes longilabius (Emerson).

TERMitidaE.-Anoplotermes banksi Emerson, A. brevipilis Emerson, Anoplotermes spp., Speculitermes silvestrii (Emerson), Microcerotermes arboreus Emerson, M. exiguus (Hagen), Crepititermes verruculosus Emerson, Termes hispaniolae (Banks), T. panamaensis (Snyder), Cavitermes tuberosus (Emerson), Neocapritermes angusticeps (Emerson), Nasutitermes costalis (Holmgren), N. ephratae (Holmgren), N. guayanae (Holmgren), N. intermedius Banks, $N$. nigriceps (Haldeman), Labiotermes labralis, subsp. boreus (Emerson), Armitermes holmgreni Snyder, Angularitermes nasutissimus (Emerson), Subulitermes baileyi (Emerson), S. parvellus (Silvestri), S. snyderi (Emerson), Subulitermes spp.

Virgin Islands, St. Croix

KALOTERMITIDAE.-Kalotermes bequaerti Snyder, $K$. incisus Silvestri, $K$. snyderi Light, Cryptotermes brevis (Walker), C. cavifrons Banks.

RHINotermitidaE.-Heterotermes convexinotatus (Snyder).

TERMITIDAE.-Termes panamaensis (Snyder), Nasutitermes costalis (Holmgren), N. ephratae (Holmgren), N. nigriceps (Haldeman).

Virgin Islands, St. Thomas

KaLotermitidae.-Kalotermes bequaerti Snyder, Cryptotermes brevis (Walker), Glyptotermes posticus (Hagen).

RHINOTERMITIDAE.-Heterotermes convexinotatus (Snyder).

TERMITIDAE.-Nasutitermes nigriceps (Haldeman).

Virgin Islands, Vieques

TERMITIDAE.-Nasutitermes nigriceps (Haldeman).

\section{Key to the Termites of the West Indies}

\section{BASED ON SOLDIERS}

Soldier absent, body worker fusiform, post clypeus strongly arched, pronotum saddle shaped, tibiae forelegs swollen.....Anoplotermes schwarzi Banks

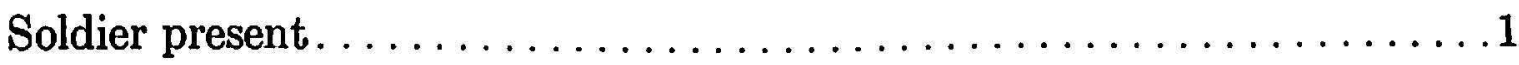

1. Mandibles degenerated into small functionless structures, with or without points, long projection in front of head $\ldots \ldots \ldots \ldots \ldots \ldots 2$ Mandibles not degenerated......................... 18 
2. Projection in front of head (labrum) long and narrow, forked at tip (minor-soldier)

Labrum wider than long (major-soldier)

Labrum longer than broad (major-soldier)

Rhinotermes marginalis (Linneaus)

Dolichorhinotermes longilabius (Emerson)

Projection in front of head not forked at tip, but prolonged into a

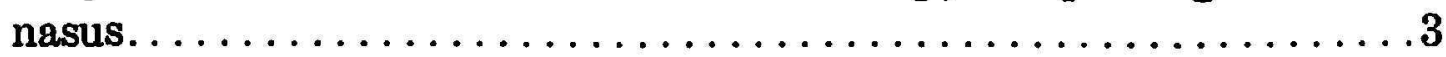

3. Head usually with conspicuous constriction in middle, base mandibles with points.............................. Head of soldier with slight constriction in middle, base mandibles without points............................ 9 Head of soldier without constriction in middle, base mandibles with

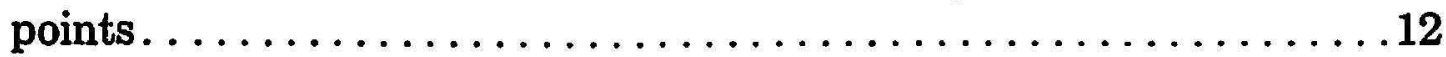

4. Third segment of antenna plainly longer than second..........5 Third segment of antenna not longer than second . . . . . . . . . 7

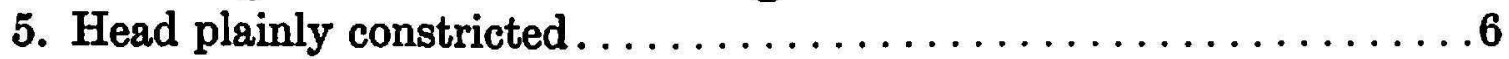

6. Head and antennae dark brown...Velocitermes antillarum (Holmgren) Head yellow-brown.................Parvitermes wolcotti (Snyder) Head but slightly constricted, yellow-brown, finely haired

Parvitermes discolor (Banks)

7. Head plainly constricted ......................... Head but slightly constricted..........Parvitermes brooksi (Snyder)

8. Head and antennae brown, femora brownish

Velocitermes toussainti (Banks) Head, antennae and legs pale yellowish. . Parvitermes flaveolus (Banks)

9. Nasus short and conical.............................

Nasus long and slender. . . . . . . . . . . . . . . . . . . 11

10. Head yellow brown, second and third segments antennae of equal length........................ Obtusitermes aequalis (Snyder) Head pale yellow, third segment antenna longer than second

11. Head convex, antennae with 11 segments

Parvitermes pallidiceps (Banks)

Subulitermes parvellus (Silvestri)

Head slightly indented in middle, antennae with 12 segments

Sublitermes snyderi (Emerson)

12. Head covered with hairs. ........................

Head covered with few hairs......................... 15

13. Head pale yellowish............Nasutitermes rippertii (Rambur)

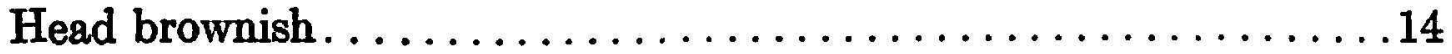

14. Head reddish or blackish-brown.... Nasutitermes nigriceps (Haldeman) 
15. Head with 10 or more hairs, nasus short

Nasutitermes guayanae (Holmgren) Head with 10 hairs, nasus long. .......Nastuitermes intermedius Banks Head with 6 or less hairs, one each side near vertex.........16

16. Four distinct hairs above base of nasus, nasus much shorter than

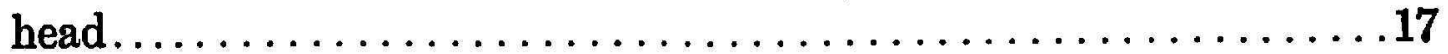
Hairs above base nasus very small, nasus nearly as long as pale head Nasutitermes hubbardi Banks

17. Head reddish-brown, nasus short, smaller

Short hairs on abdominal tergites, nasus thicker and more conical

Nasutitermes costalis (Holmgren)

Short hairs absent..............Nasutitermes ephratae (Holmgren)

18. Head longer than broad, castaneous...................19 Head short, front cavate mandibles without marginal teeth promi-

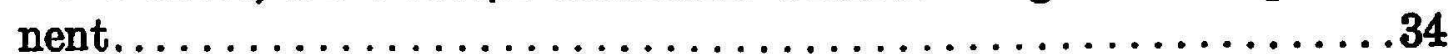

19. Mandibles with prominent marginal teeth...............20

Mandibles without prominent marginal teeth.............28

Mandibles with minute serrations

Length head 2.67-2.73 mm.......Microcerotermes arboreus Emerson Length head shorter.............Microcerotermes exiguus (Hagen)

20. Head with nasus................Armitermes holmgreni Snyder Head without nasus but with tube projecting from frontal gland

Labiotermes labralis, subsp. boreus (Emerson)

Head without nasus or tubes. . . . . . . . . . . . . . . . . .21

21. Forehead deeply lobed or steeply sloping................22

Forehead not deeply lobed or steeply sloping . . . . . . . . . 23

22. Front almost vertical, lobed

Head short, gula slender at middle

Head elongate, gula very slender at middle

Glyptotermes pubescens (Snyder)

Glyptotermes liberatus (Snyder)

Front steeply sloping, not lobed, knob on each side front head

Procryptotermes corniceps (Snyder)

23. Hind femora slender, third segment antenna not modified

Neotermes castaneus (Burm.)

Hind femora swollen, third segment antenna modified........24

24. Third segment antenna scarcely, if at all, longer than second. . . .25

Third segment antenna plainly longer than second . . . . . . . 26

25. Eyespot black, mandibles not very broad..... Kalotermes mona Banks Eyespot black, mandibles very broad..... Kalotermes cubanus Snyder Eyespot hyaline..................Kalotermes schwarzi Banks 
26. Eyespot black, gula very broad........... Kalotermes jouteli Banks Eyespot hyaline, gula slender, anterior margin of pronotum finely serrate...............................27

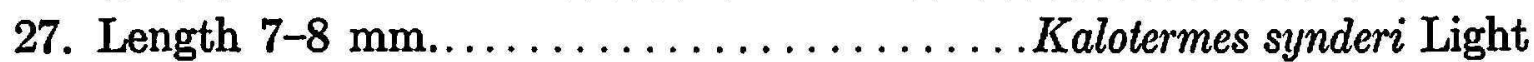
Length 5-7 mm................. Kalotermes bequaerti Snyder

28. Head with large tube in front......... Coptotermes testaceus (Linn.)

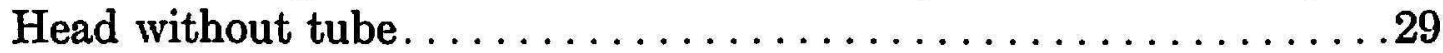

29. Mandibles long, slender, somewhat asymmetrical ........... 30 Mandibles shorter, stouter at base, more symmetrical..........32

30. Head cavate in middle.............Cavitermes tuberosus (Emerson) Head not cavate in middle.

31. Third segment antenna shorter than second

Third segment antenna longer than second

Termes panamaensis (Snyder)

Termes hispaniolae (Banks)

32. Labrum rounded at tip, head broader behind, fontanelle distinct

Prorhinotermes simplex (Hagen)

Labrum pointed at tip, head hardly broader behind, fontanelle indistinct. . . . . . . . . . . . . . . . . . . . . . . . . 33

33. Head with many hairs.............. Heterotermes tenuis (Hagen) Head with few hairs. . ..............Heterotermes cardini (Snyder) Head with very few hairs.........Heterotermes convexinotus (Snyder)

34. Head blackish, anterior tuberculate...... Cryptotermes brevis (Walker) Head blackish, anterior not tuberculate, head $1.5 \mathrm{~mm}$. long, pronotum anteriorly deeply emarginate...... Cryptotermes cavifrons Banks Head longer $1.72 \mathrm{~mm}$., pronotum anteriorly slightly emarginate

Cryptotermes havilandi (Sjostedt)

\section{BASED ON WINGED ADULTS}

1. Fontanelle or head gland absent, forewing scale usually not much longer than pronotum, branches between costal and subcostal



Fontanelle usually present, forewing scale longer than flat pronotum, no branches between costal veins . . . . . . . . . . . . . . . 8 Fontanelle usually present with plate, forewing scale shorter than saddle-shaped pronotum, no branches between costal veins......13

2. Median vein heavy, close to costal vein .................. Median vein light, free from costal veins..................6 Median vein light, usually united with subcostal vein near middle of

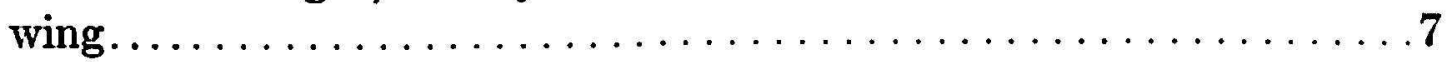

3. Wing membrane stippled, small species. . . . . . . . . . . . . 4

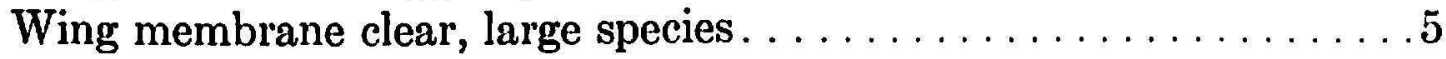


4. Head dark castaneous brown, third segment antenna shorter than second ...................... Glyptotermes pubescens (Snyder) Head and thorax red-brown...........Glyptotermes liberatus Snyder Head yellow-brown, third segment antenna longer than second

Procryptotermes corniceps (Snyder)

5. Length $21 \mathrm{~mm}$., eye large................Kalotermes mona Banks Length $18 \mathrm{~mm}$., eye smaller............Neotermes castaneus (Burm.)

6. Head and thorax yellow to castaneous, $10-16 \mathrm{~mm}$. in length Short hairs on tergites, head yellowish, length $12 \mathrm{~mm}$.

Kalotermes jouteli Banks

Long erect hairs on tergites

Ocelli oblique, head yellowish, length $15-16 \mathrm{~mm}$.

Kalotermes schwarzi Banks

Ocelli oblique, head castaneous, length $10 \mathrm{~mm}$., pulvillus between tarsal claws prominent.............Kalotermes bequaerti Snyder Ocelli round, length 11-12 mm., pulvillus absent

Kalotermes snyderi Light

7. Length $10 \mathrm{~mm} . . \ldots \ldots \ldots \ldots \ldots \ldots$ Cryptotermes brevis (Walker) Length $8.5 \mathrm{~mm} . . \ldots \ldots \ldots \ldots \ldots \ldots$. . . . . . . . . . . . Length $8 \mathrm{~mm} . . \ldots \ldots \ldots \ldots \ldots \ldots$ Cryptotermes havilandi (Sjostedt)

8. Median vein absent, wing margin not ciliate, fontanelle very distinct, yellowish-brown, length $9 \mathrm{~mm} . . . \ldots$. Prorhinotermes simplex (Hagen) Median vein present.........................

9. Wing membrane punctate, wing margins ciliate, ocelli present or absent, fontanelle indistinct or absent, in middle of head.........10 Wing membrane reticulate, ocelli present, fontanelle in front or middle head . . . . . . . . . . . . . . . . . . . . . . . . . . . . 12

10. Wing surface with many hairs, posterior margin of meso- and metanota emarginate and not covered by wing scale

Heterotermes tenuis (Hagen) $\checkmark$

Wing surface with few hairs, posterior margin of meso- and metanota convex and covered by wing scale................11

11. Cubitus vein and branches spread over more than one-half of width of wing, median near cubitus..........Heterotermes cardini (Snyder) Cubitus and branches spread over less than half of width of wing, median intermediate..........Heterotermes convexinotatus (Snyder) -

12. Head with small nasus-like process over clypeus, fontanelle near front head, 3d. joint antenna longer than 1st

3d. joint antenna shorter than 1st

Rhinotermes marginalis (Linnaeus)

Dolichorhinotermes longilabius (Emerson) 
Head without process, fontanelle in middle head

Coptotermes testaceus (Linneaus)

13. Fore tibiae with two spines........................

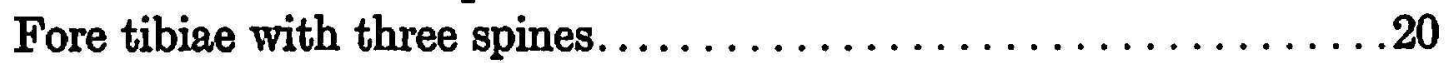

14. Left mandible with two nearly equal apical teeth, post-clypeus much shorter than half its width; pronotum weakly saddle-shaped, cubital I vein ends much before tip of wing....................15

Left mandible with first tooth longer than second, post-clypeus about as long as half its width or if shorter strongly arched, fontanelle slen-

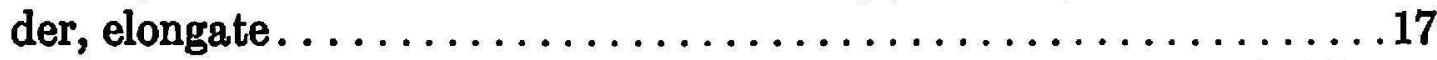
Left mandible with equal apical teeth, post-clypeus as long as half its width or if shorter, arched . . . . . . . . . . . . . . . . . 19

15. Fontanelle white, conspicuous, triangular, large eyes and ocelli, ocelli close to eyes, antennae 17-segmented, first branches cubitus heavier than others. ..........Labiotermes labralis, subsp. boreus (Emerson) Fontanelle small, round, inconspicuous, eyes and ocelli medium sized, ocelli more distant from eyes, antennae 15-segmented; first branches cubitus heavier than others..........Armitermes holmgreni Snyder Fontanelle elongate, triangular, antennae 15-segmented,

Wings and thorax blackish, eyes small not projecting, ocelli more than diameter from eye...........Nasutitermes costalis (Holmgren) Wings and thorax more yellowish

Eyes medium-sized, medium-close to margin head, ocelli close to eyes

Pronotum rounded on sides; much narrowed behind Nasutitermes ephratae (Holmgren)

Pronotum not so much narrowed behind, ocelli larger, nearer to eyes . . . . . . . . . . . Nasutitermes lividus (Burm.) Eyes very large, projecting, close to margin head, ocelli large, close to eyes, fontanelle elongate, slit-shaped

Pronotum obliquely narrowed on sides.

16. Pronotum somewhat emarginate posteriorly

Length 17-18 mm.............. Nasutitermes nigriceps (Hald.) Length $16-17 \mathrm{~mm} . . \ldots \ldots \ldots \ldots \ldots \ldots$ Nasutitermes rippertii (Rbr.) Length 13-15 mm..............Nasutitermes intermedius Banks Pronotum markedly marginate posteriorly, fontanelle not so elongate, antennae 16-segmented........ Nasutitermes guayanae (Holmg.)

17. Third segment antenna not longer than second, mandibles short and

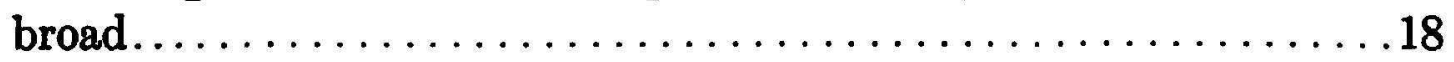
Third segment antenna very short, mandibles long, fontanelle a raised spot, meso- and meta-nota notched, head densely hairy, $2 \mathrm{~mm}$. broad......................Anoplotermes schwarzi Banks 
Head less densely hairy, less broad

Anoplotermes meridianus Emerson

18. Eye large, prominent, second segment antenna longer than third, antenna with 14 segments. .........Subulitermes snyderi (Emerson) Eye fairly large, not prominent, second segment antenna equal to third, antenna with 16 segments.... Subulitermes parvellus (Silvestri)

19. Third segment antenna plainly longer than second, mandibles short and broad, eye small, projecting, head arched behind

Antennae with 12-13 segments, head brownish..... Parvitermes spp. Antennae with 16 segments, head darker

Velocitermes antillarum (Holmgren)

20. Mandibles with very large first tooth, post-clypeus large, arched, about as long as half its breadth, fontanelle, eyes and ocelli small, pronotum flat, cubital vein ends near tip of wing...........21 Mandibles with subequal apical teeth, head oval, parallel sided; pronotum saddle-shaped, eyes and ocelli small, fontanelle indistinct....23

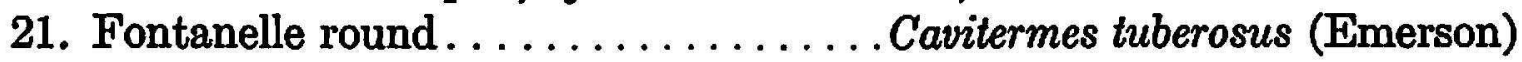
Fontanelle slit-shaped . . . . . . . . . . . . . . . . . . 22

22. Length $8 \mathrm{~mm}$., wing scale as long as pronetum

Length shorter, wing scale shorter than pronotum

Termes hispaniolae (Banks)

Termes panamaensis (Synder)

23. Length 9 mm.,..............Microcerotermes arboreus Emerson Length shorter................. Microcerotermes exiguus (Hagen)

\section{SUMMARY}

Many of the termites of the West Indies are endemic, and so far as known, have a very limited distribution, often recorded from only a few islands. Kalotermes mona Banks is endemic to Mona Island and is found nowhere else. By contrast, many of the termites occuring in Cuba are also found in the United States, those of Jamaica in Panamá and Central America, and those of Trinidad in South America. The truly tropicosmopolitan Cryptotermes brevis (Walker) and Nasutitermes costalis (Holmgren) readily become established in new localities, islands or countries in which the environmental conditions are at all suitable.

The comparatively large island of Trinidad, close to the mainland of South America and mostly with a continental fauna, has the largest number of species of termites of any of the West Indies: 31 . The island of Curaçao, also near the coast of South America, but small and arid has but 2, and none is recorded from Aruba, Margarita or the other smaller islands off the north coast of Venezuela. The comparatively small island of Tobago, northeast from Trinidad, has 8 species of termites recorded, and Barbados 
7, but none is listed from St. Vincent, and most of the other Lesser Antilles have at most but 3 or 4 . None is reported from Nevis, Saba, or St. John, but St. Croix of the U. S. Virgin Islands has 10, St. Thomas 5, and Culebra and Vieques 1 each. Fifteen species of termites are recorded from Puerto Rico, and 4 from Mona. In the large island of Hispaniola, only 8 species of termites are recorded from the eastern portion: the Dominican Republic, while 18 are known from Haiti. Sixteen species of termites are known in Jamaica, and 9 from the widely dispersed islands of the Bahamas. The large island of Cuba, closest of any to continental North America, has 22; the comparatively minute, and most distant Bermuda has 4.

All recorded species may be identified by means of keys based on the characters of the soldiers, or of the winged adults.

\section{RESUMEN}

Muchos de los comejenes de las Antillas son endémicos. Hasta donde se sabe, tienen una distribución muy limitada por lo que sólo existen récords de su presencia en muy pocas islas. A modo de contraste, muchos de los comejenes de Cuba también se pueden encontrar en los Estados Unidos; los de Jamaica en Panamá y los de Trinidad en Sur América. Los verdaderos cosmopolitas del Trópico, el Cryptotermes brevis (Walker) y el Nasutitermes costalis (Holmgren) se establecen fácilmente en nuevas localidades, islas o países donde las condiciones del medio ambiente les son favorables.

La comparativamente extensa isla de Trinidad, cerca del continente de Sur América cuya fauna es casi la misma que la continental, tiene el mayor número de comejenes entre todas las Antillas, el cual asciende a 31 . La isla de Curazao, también cerca de la costa suramericana, y con un área pequeña $\mathrm{y}$ árida sólo tiene 2 especies. Aruba no tiene, tampoco Margarita ni las otras islas más pequeñas cerca de la costa norte de Venezuela. La relativamente pequeña isla de Tobago, al noroeste de Trinidad tiene 8 especies de comején, Barbada tiene 7, pero según la lista St. Vincent no cuenta con especie alguna. La mayoría de las Antillas Menores sólo tiene 3 ó 4. Nevis, Saba y St. John no tienen, pero St. Croix en las Islas Vírgenes Americanas informa tener $10 \mathrm{y}$ St. Thomas 5. Culebras y Vieques informan 1 especie cada una.

Hay en récord 15 especies de comején en Puerto Rico. La Mona tiene 4. La Española, isla de gran extensión territorial, tiene 8 especies en su región oriental ocupada por la República Dominicana, mientras que en Haití hay 18. Los récords señalan que en Jamaica hay 16 especies de comején y 9 especies están dispersas entre las islas Bahamas. La gran isla de Cuba, la más cercana al continente de Norte America tiene 22 especies, la comparativamente diminuta Bermuda tiene 4.

Todos las especies en récord se pueden identificar por medio de claves, las cuales se basan en los caracteres de los soldados, o en los adultos alados. 